\title{
Artificial Placenta: A Novel Approach for Preterm Neonates: Review of Literature
}

\section{Kumar $\mathbf{N}^{*}$}

Associate Professor, Department of Obstetrics and Gynaecology, Maharishi Markandeshwar Institute of Medical Sciences and Research, India

*Corresponding author: Kumar N, MD, Associate Professor, Department of Obstetrics and Gynaecology, Maharishi Markandeshwar Institute of Medical Sciences and Research, Mullana, Ambala, Haryana, India, E-mail: drnainakumar@gmail.com

Citation: Kumar N (2017) Artificial Placenta: A Novel Approach for Preterm Neonates: Review of Literature. J Gynecol Res 3(1): 101. doi: 10.15744/2454-3284.3.101

Received Date: January 08, 2017 Accepted Date: May 22, 2017 Published Date: May 23, 2017

\begin{abstract}
Preterm birth is one of the most common causes of neonatal morbidity and mortality worldwide. Furthermore the mortality and morbidity is very high in extremely preterm infants born before 28 completed weeks of gestation or those with extremely low birth weight for gestational age $(<1000 \mathrm{~g})$. One of the major causes for such a high morbidity and mortality in such infants is respiratory insufficiency, due to poor lung maturity. To overcome these problems and to reduce overall burden of morbidity and mortality associated with immature pulmonary development in preterm neonates, the concept of "artificial placenta" came into picture, which is basically a pump less lung assisted device connected to umbilical vessels that helps in gaseous exchange in neonates with severe respiratory insufficiency. Hence, the present study will allow us to throw light on recent developments in the field of use of artificial placenta technology for saving many more lives of preterm, low birth weight neonates. Method: Literature regarding preterm births and use of artificial placenta for saving lives was searched from various English language journals, Government sites, WHO, UNICEF data, published peer-reviewed articles on PubMed, MEDLINE, Embase and Google Scholar till 2017.
\end{abstract}

Keywords: Artificial Placenta; Low birth weight; Preterm births; Neonates; Respiratory insufficiency

\section{Introduction}

The artificial placenta was designed to mimic working of natural placenta in-utero and to allow partial fetal circulation and placental gaseous exchange while directly connected to umbilical vessels [1]. The first attempts to develop an artificial placenta as an effective alternation to provide respiratory support for newborns, especially preterm were made in mid-20th century [2]. Since, then for many decades it has fascinated the imaginations of researchers as an alternative approach to help extremely preterm infants (EPIs) born less than 28 weeks of gestation to overcome respiratory insufficiency [3,4].

An era came when the treatment of premature infants improved with newer advances like use of prenatal maternal steroids, exogenous surfactant and more advanced modes of ventilation [5,6], leading to loss of interest in artificial placenta, but despite all these advances the final outcome of extremely preterm infants remained unchanged. Hence, there was resurge of interest in development of artificial placentas for improving overall survival of extremely preterm infants [7-9].

Till date many attempts have been made to develop newer and better models of Artificial Placenta that can help save lives of many more preterm infants.

\section{Preterm Births, Its Burden and Complications}

Preterm birth is when babies are born before 37 completed weeks of gestation. Annually around 15 million babies are born before 37 completed weeks and account for nearly 1 million deaths of children below 5 years of age [10]. Hence, prematurity is one of the leading causes of perinatal and neonatal morbidity and mortality all over the world [11]. The high morbidity and mortality associated with prematurity and low birth weight is due to high vulnerability of such babies to complications like respiratory insufficiency or distress, feeding problems, increased risk of sepsis, jaundice and temperature instability [12-14].

An estimated 12.9 million births, or $9.6 \%$ of total births worldwide, were preterm in 2005 . Of these 11 million (85\%) preterm births occurred in Africa and Asia, 0.5 million each in Europe and North America (excluding Mexico) and 0.9 million in Latin America and Caribbean [15]. On the other hand the recent Indian data reveal that annually around 3,341,000 babies are born preterm $(<37$ weeks) with an annual preterm birth rate of $13 \%$. Of these around 165,800 are born at $<28$ completed weeks of gestation. Also it 
was reported that in India an estimated 361,600 children under five die due to direct complications of prematurity [16,17].

Worldwide, preterm deaths constitute $28 \%$ of total 4 million annual new born deaths with an estimated $99 \%$ of these deaths occurring in developing countries [18]. Furthermore, around $45 \%$ to $50 \%$ of preterm births are idiopathic, 30\% due to preterm prelabour rupture of membranes (PPROM) and about 15-20\% are medically indicated or elective preterm deliveries [15,19,20].

At the extreme end of spectrum of prematurity are extremely preterm infants (EPI) born before 28 weeks of gestation [10] with very low birth weight $(<1000 \mathrm{~g})$ and ultra extremely preterm infants or "micro-preemies" weighing $<400 \mathrm{~g}$ and $<600 \mathrm{~g}$ at 23 and 25 weeks respectively [21], which are considered in "grey-zone" as they have a very high mortality rate due to poorly developed vital organs necessary for sustaining life [3]. These neonates, especially those in grey-zone are at increased risk of developing respiratory failure and ultimately long-term morbidity and mortality [22]. Despite of advances in field of neonatology and development of exogenous surfactant replacement therapy and low-pressure ventilator techniques, iatrogenic injury to immature lungs is still common [23]. Even conventional extracorporeal life support or extracorporeal membrane oxygenation (ECLS/ECMO) causes severe damage to immature lungs, and is contraindicated in extremely low gestational age newborns (ELGANs) [24]. Hence, the best way to save such neonates is through use of artificial placenta that helps in maintaining fetal circulation and bypassing lungs, thus obviating the need for mechanical ventilation and allowing the lungs to mature [4].

\section{Role of Artificial Placenta in Management of Prematurity}

An artificial placenta using extracorporeal life support (ECLS) has been investigated as one of the most effective way of treating respiratory insufficiency in ELGANs [25]. Respiratory distress or insufficiency is one of the leading causes of neonatal longterm morbidity and mortality, especially in EPI and those born with very low birth weight [26]. It was reported that annually an estimated 24,000 new born infants suffer from respiratory distress in the United States, of which one third are born before 28 weeks of gestation [27].

The most appealing solution for correcting respiratory insufficiency in EPI and to reduce the overall mortality rates associated with it, is by returning them to a uterine-like environment by using "artificial placenta", which is a pump-less lung assisted device connected to the umbilical vessels [1].

Artificial placenta works on same principle as a normal placenta works in-utero, serving as an interface between maternal and fetal circulations thereby providing fetal nutrient, metabolite elimination, and exchange of gases [1]. In preterm infants, especially with severe respiratory insufficiency, the artificial placenta helps in gaseous exchange until the baby recovers from its initial cause of lung failure in its early neonatal period. Hence, artificial placenta which is connected to umbilical vessels, similar to natural placenta, works in parallel with systemic circulation [1]. It is basically a pump-less device which is driven by arteriovenous pressure difference alone. In addition to forming partial fetal circulation, the artificial placenta also helps in perfusion and ventilation of lungs [1]. It significantly helps in gaseous exchange of newborn, but, since the residual lung function is still present, artificial placenta does not meet the total body needs of newborn [28,29]. Hence, less amount of blood flow is required to circulate through extracorporeal device, thereby decreasing the risk of high-output cardiac failure [1]. Figure 1 depicts the diagrammatic representation of artificial placenta [30].

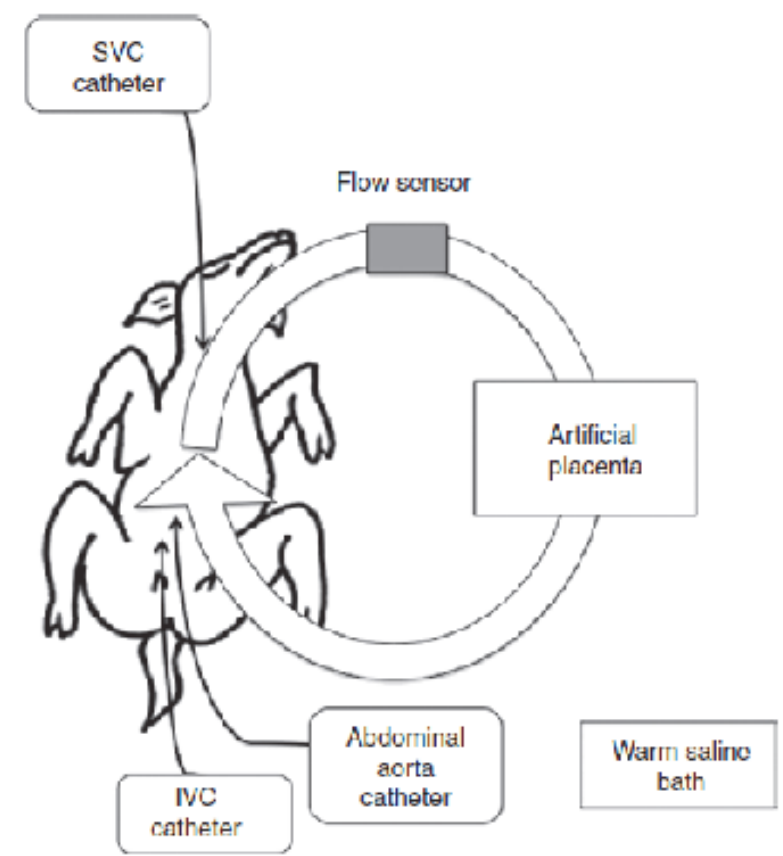

Figure 1: Artificial placenta. Both fetus and circuit were submerged and warmed in saline bath kept at $39^{\circ} \mathrm{C}$ 
Furthermore, the artificial placenta must mimic the functioning of native placenta, with the aim that it should retain fetal circulation, thereby bypassing the developing lungs [3]. This can be achieved when artificial placenta maintains; fetal circulation without affecting patency of major fetal shunts, improves blood oxygenation and corrects haemoglobin saturation without causing lung inflation, leads to hemodynamic stability including, heart rate and blood pressure in response to fetal states, maintains fluid balance and electrolyte composition to ensure proper hydration or removal of excess fluid, enhances kidney functions for removal of metabolic nitrogenous waste and finally provides endocrine support [3]. Hence, artificial placenta can help in decreasing the overall mortality of extremely preterm infants and the associated short and long term morbidities in near future.

\section{Conclusion}

Artificial Placenta is an effective option for saving lives of preterm and extremely preterm infants. It helps in maintaining fetal circulation, gaseous exchange and hemodynamic stability in preterm infants. Artificial placenta has the potential in near future to completely change the paradigm of treating prematurity. Newer research are still under trial for developing major components of artificial placenta like oxygenator, vascular access via umbilical vessels, flow control, hemocompatibility in extracorporeal circuits for further improving its working and compatibility.

\section{Acknowledgement}

I acknowledge and thank Dr. Namit Kant Singh for his kind support and advice.

\section{References}

1. Rochow N, Chan EC, Wu WI, Selvaganapathy PR, Fusch G, et al. (2013) Artificial placenta--lung assist devices for term and preterm newborns with respiratory failure. Int J Artif Organs 36: 377-91.

2. Enhorning G, Westin B (1954) Experimental studies of the human fetus in prolonged asphyxia. Acta Physiol Scand 31: 359-75.

3. Bird SD (2017) Artificial placenta: Analysis of recent progress. Eur J Obstet Gynecol Reprod Biol 208: 61-70.

4. Gray BW, El-Sabbagh A, Zakem SJ, Koch KL, Rojas-Pena A, et al. (2013) Development of an artificial placenta V: 70 h veno-venous extracorporeal life support after ventilatory failure in premature lambs. J Pediatr Surg 48: 145-53.

5. Liggins GC, Howie RN (1972) A controlled trial of antepartum glucocorticoid treatment for prevention of the respiratory distress syndrome in premature infants. Pediatrics 50: 515-25.

6. Butler WJ, Bohn DJ, Bryan AC, Froese AB (1980) Ventilation by high-frequency oscillation in humans. Anesth Analg 59: 577-84.

7. Kuwabara Y, Okai T, Imanishi Y, Muronosono E, Kozuma S, et al. (1987) Development of extrauterine fetal incubation system using extracorporeal membrane oxygenator. Artif Organs 11: 224-7.

8. Yasufuku M, Hisano K, Sakata M, Okada M (1998) Arterio-venous extracorporeal membrane oxygenation of fetal goat incubated in artificial amniotic fluid (artificial placenta): influence on lung growth and maturation. J Pediatr Surg 33: 442-8.

9. Awad JA, Cloutier R, Fournier L, Major D, Martin L, et al. (1995) Pumpless respiratory assistance using a membrane oxygenator as an artificial placenta: a preliminary study in newborn and preterm lambs. J Invest Surg 8: 21-30.

10. Blencowe H, Cousens S, Oestergaard MZ, Chou D, Moller AB, et al. (2012) National, regional, and worldwide estimates of preterm birth rates in the year 2010 with time trends since 1990 for selected countries: a systematic analysis and implications. Lancet 379: 2162-72.

11. Kinney MV, Lawn JE, Howson CP, Belizan J (2012) 15 million preterm births annually: what has changed this year? Reprod Health 9: 28.

12. Escobar GJ, McCormick MC, Zupancic JA, Coleman-Phox K, Armstrong MA, et al. (2006) Unstudied infants: outcomes of moderately premature infants in the neonatal intensive care unit. Arch Dis Child Fetal Neonatal Ed 91: F238-44.

13. Kinney HC (2006) The near-term (late preterm) human brain and risk for periventricular leukomalacia: a review. Semin Perinatol 30: 81-8.

14. Wang ML, Dorer DJ, Fleming MP, Catlin EA (2004) Clinical outcomes of near-term infants. Pediatrics 114: 372-6.

15. Beck S, Wojdyla D, Say L, Betran AP, Merialdi M, et al. (2010) The worldwide incidence of preterm birth: a systematic review of maternal mortality and morbidity. Bull World Health Organ 88: 31-8.

16. UNICEF (2014) State of the World's Children 2015. New York: UNICEF.

17.WHO, UNICEF, UNFPA (2014) The World Bank, United Nations Population Division. Trends in Maternal Mortality: 1990 to 2013. Geneva, WHO.

18. Federal Ministry of Health (2011) Saving Newborn Lives in Nigeria. Newborn Health I the Context of the Integrated Maternal, Newborn and Child Health Strategy. Revised 2nd Edition. with time trends since 1990 for selected countries: a systematic analysis and implications. Lancet 379: 2162-72.

19. Pennell CE, Jacobsson B, Williams SM, Buus RM, Muglia LJ, et al. (2007) PREBIC Genetics Working Group., Relton C. Genetic epidemiologic studies of preterm birth: guidelines for research. Am J Obstet Gynecol 196: 107-18.

20. Haas DM (2006) Preterm birth in clinical evidence. London: BMJ Publishing Group.

21. Dobbins TA, Sullivan EA, Roberts CL, Simpson JM (2012) Australian national birth weight percentiles by sex and gestational age, 1998-2007. Med J Aust 197: 291-4.

22. Ali K, Greenough A (2012) Long-term respiratory outcome of babies born prematurely. Ther Adv Respir Dis 6: 115-20.

23. Sweet DG, Carnielli V, Greisen G, Hallman M, Ozek E, et al. (2010) European Association of Perinatal Medicine. European consensus guidelines on the management of neonatal respiratory distress syndrome in preterm infants - 2010 update. Neonatology 97: 402-17.

24. Skinner SC, Hirschl RB, Bartlett RH (2006) Extracorporeal life support. Semin Pediatr Surg 15: 242-50.

25. Davis RP, Bryner B, Mychaliska GB (2014) A paradigm shift in the treatment of extreme prematurity: the artificial placenta. Curr Opin Pediatr 26: 370-6.

26. Rodriguez RJ, Martin RJ, Fanaroff AA (2002) Respiratory distress syndrome and its management. In: Fanaroff AA, Martin RJ, eds. Fanaroff and Martin's Neonatal-Perinatal Medicine: Diseases of the Fetus and Infant. 7th ed. St. Louis, MO: Mosby 1001-11. 
27. Hermansen CL, Lorah KN (2007) Respiratory distress in the newborn. Am Fam Physician 76: 987-94.

28. Reoma JL, Rojas A, Kim AC, Khouri JS, Boothman E, et al. (2009) Development of an artificial placenta I: pumpless arterio-venous extracorporeal life support in a neonatal sheep model. J Pediatr Surg 44: 53-9.

29. Bauer J, Maier K, Hellstern G, Linderkamp O (2003) Longitudinal evaluation of energy expenditure in preterm infants with birth weight less than 1000 g. Br J Nutr 89: 533-7.

30. Miura Y, Matsuda T, Funakubo A, Watanabe S, Kitanishi R, et al. (2012) Novel modification of an artificial placenta: pumpless arteriovenous extracorporeal life support in a premature lamb model. Pediatr Res 72: 490-4.

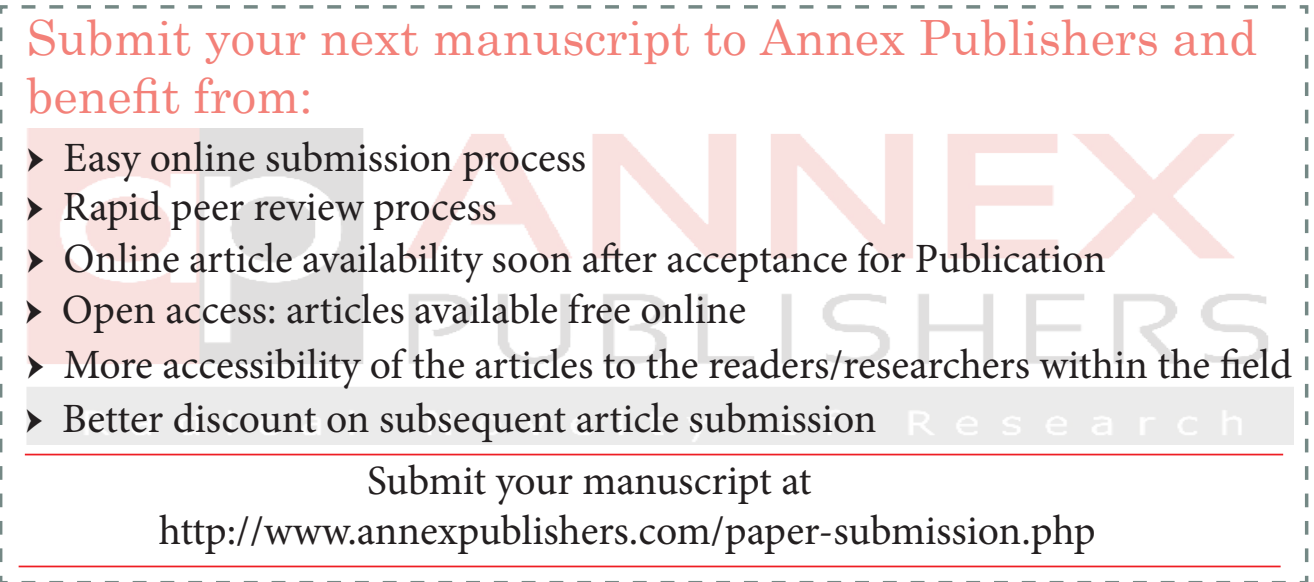

